\title{
A mannose-sensitive haemagglutinin (MSHA)-like pilus promotes attachment of Pseudoalteromonas tunicata cells to the surface of the green alga U/va australis
}

\author{
Doralyn S. Dalisay, ${ }^{1}$ Jeremy S. Webb, ${ }^{2,3} \dagger$ André Scheffel, ${ }^{4}$ \\ Charles Svenson, ${ }^{2}$ Sally James, ${ }^{2,3}$ Carola Holmström, ${ }^{2,3}$ Suhelen Egan ${ }^{2,3}$ \\ and Staffan Kjelleberg ${ }^{2,3}$ \\ ${ }^{1}$ Department of Chemistry and Biochemistry, University of California, San Diego, 9500 Gilman \\ Drive, La Jolla, CA 92093, USA \\ ${ }^{2,3}$ School of Biotechnology and Biomolecular Sciences ${ }^{2}$ and Centre for Marine Biofouling and \\ Bio-innovation ${ }^{3}$, University of New South Wales, Sydney, NSW 2052, Australia \\ ${ }^{4}$ Max-Planck-Institute for Marine Microbiology, Celsiusstraße 1 28359, Bremen, Germany
}

Correspondence

Staffan Kjelleberg

s.kjelleberg@unsw.edu.au

Received 25 May 2006

Revised 10 July 2006

Accepted 12 July 2006
This study demonstrates that attachment of the marine bacterium Pseudoalteromonas tunicata to the cellulose-containing surface of the green alga Ulva australis is mediated by a mannose-sensitive haemagglutinin (MSHA-like) pilus. We have identified an MSHA pilus biogenesis gene locus in $P$. tunicata, termed msh/1/2JKLMNEGFBACDOPQ, which shows significant homology, with respect to its genetic characteristics and organization, to the MSHA pilus biogenesis gene locus of Vibrio cholerae. Electron microscopy studies revealed that $P$. tunicata wild-type cells express flexible pili peritrichously arranged on the cell surface. A P. tunicata mutant (SM5) with a transposon insertion in the $m s h J$ region displayed a non-piliated phenotype. Using SM5, it has been demonstrated that the MSHA pilus promotes attachment of $P$. tunicata wild-type cells in polystyrene microtitre plates, as well as to microcrystalline cellulose and to the living surface of $U$. australis. $P$. tunicata also demonstrated increased pilus production in response to cellulose and its monomer constituent cellobiose. The MSHA pilus thus functions as a determinant of attachment in $P$. tunicata, and it is proposed that an understanding of surface sensing mechanisms displayed by $P$. tunicata will provide insight into specific ecological interactions that occur between this bacterium and higher marine organisms.

\section{INTRODUCTION}

Marine surfaces are colonized by a diversity of microorganisms and sessile marine organisms collectively known as biofouling communities. The biofouling process is initiated by the attachment of bacteria to a surface followed by the settlement and adherence of diatoms, free-swimming algal spores and invertebrate larvae (Bryers \& Characklis, 1982). Some sessile higher organisms employ chemical defences against biofouling through the production of

tPresent address: School of Biological Sciences, University of Southampton, Southampton SO16 7PX, UK.

Abbreviations: CLSM, confocal laser scanning microscopy; GFP, green fluorescent protein; MSHA, mannose-sensitive haemagglutinin; RFP, red fluorescent protein; TEM, transmission electron microscopy.

The GenBank/EMBL/DDBJ accession numbers for the sequences reported in this paper are AY695819 and ZP_01133305ZP_01133315. secondary metabolites that inhibit the development and formation of a biofouling community (Harrison, 1992; Mary et al., 1993; Maximilien et al., 1995). For example, furanones produced by the red alga Delisea pulchra have been reported to inhibit the settlement of common fouling organisms (de Nys et al., 1995). For marine organisms without intrinsic defence mechanisms, it has been proposed that protection against fouling is maintained by the secondary metabolites produced by surface-associated bacteria (Egan et al., 2001a, b; Holmström et al., 1992, 1996, 1998; Holmström \& Kjelleberg, 1999; James et al., 1996).

Many species of the genus Pseudoalteromonas have been found to produce bioactive compounds against different classes of fouling organisms and are frequently found in association with the surfaces of living marine eukaryotes (Holmström \& Kjelleberg, 1999). A well-studied surfaceassociated bacterium is Pseudoalteromonas tunicata. This 
green-pigmented bacterium was first isolated from the surface of a tunicate, Ciona intestinalis, in Sweden (Holmström et al., 1998) and later from a green alga, Ulva australis (formally Ulva lactuca), in Australian waters (Egan et al., 2001a). P. tunicata produces a number of extracellular bioactive compounds, each of which has a specific inhibitory activity against target organisms such as algal spores, fungi, invertebrate larvae or bacteria (Egan et al., 2001b; Holmström et al., 1992, 2002; James et al., 1996). While several studies have addressed the production of bioactive compounds, no data have so far been reported on the means by which $P$. tunicata colonizes surfaces, including those of higher marine organisms. Investigation of such mechanisms will contribute to a greater understanding of the ecology of $P$. tunicata and its interaction with marine surfaces.

The successful colonization of surfaces by bacteria is often mediated by cell-surface appendages such as pili and flagella. For example, the attachment of Escherichia coli to abiotic surfaces is promoted by the presence of both type 1 pili and flagella (O'Toole \& Kolter, 1998). In Pseudomonas aeruginosa, flagella are believed to be important for initial attachment to a surface, while type 4 pili promote the formation of microcolonies on the surface (O'Toole \& Kolter, 1998). It has also been reported that type 4 pili mediate attachment of pathogenic bacteria such as Neisseria gonorrhoeae (Morand et al., 2001) and Pseudomonas aeruginosa (Zolfaghar et al., 2003) to host epithelial cells. The adherence of Vibrio cholerae to environmental surfaces is directly associated with the presence of the mannosesensitive haemagglutinin (MSHA) pilus, which belongs to a family of type 4 pili (Marsh \& Taylor, 1999). In V. cholerae, the MSHA pilus has been demonstrated to play a direct role in both colonization and subsequent biofilm formation on abiotic as well as biotic surfaces (Chiavelli et al., 2001; Watnick et al., 1999; Watnick \& Kolter, 1999).

Here we describe a non-piliated mutant of $P$. tunicata (SM5) that carries a transposon insertion in an ORF termed $m s h J$, with high homology to the $V$. cholerae $m s h J$, a gene encoding a MSHA pilus biogenesis protein. DNA sequencing upstream and downstream of $m s h J$ revealed the presence of a gene locus with 17 ORFs that are homologous to the MSHA pilus biogenesis gene locus of $V$. cholerae, and we propose that this gene locus is involved in the assembly and transport of an MSHA pilus in $P$. tunicata. Here we demonstrate a role for the $P$. tunicata MSHA pilus in attachment to abiotic, as well as biotic (U. australis) surfaces, and propose that $P$. tunicata demonstrates surface sensing mechanisms as revealed by increased pilus production in the presence of cellulose, one of the major surface polymers of U. australis.

\section{METHODS}

Bacterial strains, plasmids and culture conditions. $P$. tunicata wild-type was maintained on the complex marine medium VNSS (Marden et al., 1985). For attachment assays, P. tunicata was grown in nine salt solution (NSS), $\mathrm{pH} 7 \cdot 0$, or Marine Minimal Medium (3M) (Neidhardt et al., 1974), each medium containing either $0.05 \%(\mathrm{w} / \mathrm{v})$ glucose, $0.25 \%$ microcrystalline cellulose (Avicel; Fluka) or $0 \cdot 1 \%$ cellobiose (Sigma) as the sole source of carbon. $P$. tunicata SM5 was isolated from a $P$. tunicata mini-Tn 10 transposon library (generated according to the method of Egan et al., 2002a) and was selected on the basis of reduced attachment to polystyrene microtitre plates according to the method of O'Toole \& Kolter (1998). The P. tunicata SM5 strain was grown on VNSS agar plates containing $100 \mu \mathrm{g}$ streptomycin $\mathrm{ml}^{-1}$ and $85 \mu \mathrm{g}$ kanamycin $\mathrm{ml}^{-1}$.

P. tunicata wild-type cells were provided with a green fluorescent protein (GFP) colour tag by transconjugation using the constitutive GFP expression plasmid pCJS10. This plasmid contains the gfpmut3 gene (Cormack et al., 1996) on an RSF1010 backbone from broad-hostrange vector pHRP304 (Bagdasarian et al., 1981). In addition, a red fluorescent protein (RFP) colour tag was provided to SM5 cells using the pCJS10-derived plasmid pCJS10R. This plasmid contains the RFP gene dsred (Clontech) in place of gfpmut 3 on pCJS10 (Rao et al., 2005). Triparental conjugations were carried out as described previously for $P$. tunicata (Egan et al., 2002a) and labelled transconjugants were grown on VNSS agar plates containing $15 \mu \mathrm{g}$ chloramphenicol $\mathrm{ml}^{-1}$ and $100 \mu \mathrm{g}$ streptomycin $\mathrm{ml}^{-1}$. GFP- and RFP-labelled strains showed bright fluorescence after overnight culture and we observed no differences in the growth rate or surface attachment properties of the labelled strains versus the unlabelled parent strain (data not shown).

Panhandle PCR, DNA sequencing and sequence analysis. To obtain sequence information from the genes disrupted by the miniTn 10 transposon in the SM5 mutant, panhandle PCR was carried out as described previously using adaptor-specific primer AP1 (5' GGATCCTAATACGACTCACTATAGGGC-3') and transposon-specific primers Tn10C (5'-GCTGACTTGACGGGACGGCG- $\left.3^{\prime}\right)$ and Tn10D (5'-CCTCGAGCAAGACGTTTCCCG-3') (Egan et al., 2002a, b). Panhandle PCR products were visualized in $1 \%$ agarose gel and purified using a PCR purification kit (Qiagen), according to the manufacturer's instructions. PCR products were sequenced using transposon-specific primers Tn10C and Tn10D and a primer walking strategy. For sequencing, between 50 and $100 \mathrm{ng}$ doublestranded template DNA, $1 \mu \mathrm{l}$ specific primer (10 pmol), $4 \mu \mathrm{l}$ CSA buffer and $4 \mu \mathrm{l}$ BigDye terminator cycle sequencing reaction mix (Applied Biosystems) were mixed in a final volume of $20 \mu \mathrm{l}$. Amplification of DNA was conducted using the following parameters: $94{ }^{\circ} \mathrm{C}$ for the initial denaturation step which was followed by 25 cycles of $94{ }^{\circ} \mathrm{C}$ for $10 \mathrm{~s}, 50{ }^{\circ} \mathrm{C}$ for $5 \mathrm{~s}$ and $60^{\circ} \mathrm{C}$ for $4 \mathrm{~min}$. After cycling, the sequencing mixture was cleaned and purified using a butanol purification protocol (Tillett \& Neilan, 1999). Separation of sequencing products was performed on an ABI 377 DNA sequencing system at the Sydney University Prince Alfred Macromolecular Analysis Centre (SUPAMAC). The DNA sequence electropherograms were analysed with ABI-PRISM software. Multiple sequence alignments were performed using the Staden Package System (Medical Research Council - Laboratory of Molecular Biology, University of Cambridge). The complete consensus DNA sequence was compared with sequences in the GenBank database using the BLAST-search algorithm (Altschul et al., 1990) and ORFs were defined using the ORF finder program made available through the National Center for Biotechnology Information (NCBI) website (www.ncbi.nlm.nih.gov). Promoter prediction was performed using the neural network promoter prediction tool (Reese, 2001) available though the Berkley Drosophila genome project website (www. fruitfly.org/seq_tools/promoter.html). The GCG software package provided by the Australian National Genomic Information Service (ANGIS) website (www.angis.org.au/WebANGIS/) and the ExPASy (Expert Protein Analysis System) site (http://expasy.proteome. org.au/index.html) of the Swiss Institute of Bioinformatics (SIB) 
were also used for sequence analysis. Additional sequence information was obtained by analysis of the draft genome sequence for $P$. tunicata using the BLAST-search algorithm (Altschul et al., 1990).

Transmission electron microscopy (TEM). Cell surface morphology of $P$. tunicata wild-type and SM5 strains was examined using TEM. Bacterial cells were grown on VNSS plates for $24 \mathrm{~h}$ (equivalent to the stationary phase of growth) and colonies were gently resuspended in PBS, pH 7.4 $\left(1^{-1}: 8.00 \mathrm{~g} \mathrm{NaCl}, 0.20 \mathrm{~g} \mathrm{KCl}, 1.44 \mathrm{~g}\right.$ $\mathrm{Na}_{2} \mathrm{HPO}_{4}, 0.24 \mathrm{~g} \mathrm{KH}_{2} \mathrm{PO}_{4}$ ) to a concentration of $10^{6}$ cells $\mathrm{ml}^{-1}$. Alternatively, bacterial cells were grown in static conditions in NSS liquid medium containing either glucose, cellobiose or cellulose as the sole carbon source. Carbon and Formvar-coated copper grids were placed on a drop of cell suspension for $5 \mathrm{~min}$ and then negatively stained with $2 \%$ phosphotungstic acid for $30 \mathrm{~s}$. The cells were examined using a Hitachi H7000 transmission electron microscope.

Haemagglutination assay. Haemagglutination assays were performed as described by Gardel \& Mekalanos (1996). Bacterial strains were grown in VNSS medium at $28^{\circ} \mathrm{C}$ under static conditions and assayed during both exponential and stationary growth phases. Briefly, the cells were washed twice and resuspended in KrebsRinger solution (KRT). An initial concentration of $10^{10} \mathrm{cells} \mathrm{ml}^{-1}$ was serially diluted in 96-well microtitre plate with each well containing $100 \mu \mathrm{l} 3 \%(\mathrm{v} / \mathrm{v})$ washed horse erythrocyte suspension. The mixture was incubated at room temperature for $30 \mathrm{~min}$ and scored for haemagglutination. $V$. cholerae strain M1615 was used as a positive control. To determine if pili are sensitive to the presence of mannose during the haemagglutination assay, $50 \mu 11 \% \alpha$-methyl-Dmannoside (a non-metabolizable derivative of mannose) were added to the haemagglutination mixture. The haemagglutination assay for each strain was carried out in three replicates.

Attachment assays. The attachment of the P. tunicata wild-type and the SM5 mutant strains to abiotic surfaces was tested by modifying an attachment assay described by O’Toole \& Kolter (1998). Bacterial isolates were grown under static conditions at $28^{\circ} \mathrm{C}$ on VNSS medium and harvested after $24 \mathrm{~h}$. The cell suspension was centrifuged $(6000 \mathrm{~g}, 5 \mathrm{~min})$, washed twice and resuspended in $10 \mathrm{ml}$ PBS, $\mathrm{pH} 7 \cdot 4$, to an $\mathrm{OD}_{600}$ of $0 \cdot 6-0 \cdot 7$. Aliquots $(1 \mathrm{ml})$ of cell suspension were added to wells of a 24 -well polystyrene microtitre plate. The plate was shaken slowly for $1 \mathrm{~h}$ at room temperature. Wells were then washed twice using sterile distilled water and air-dried for $45 \mathrm{~min}$. The attached cells were fixed at $80{ }^{\circ} \mathrm{C}$ for $30 \mathrm{~min}$ and stained with $0 \cdot 1 \%$ crystal violet for $45 \mathrm{~min}$. The cells were destained with $95 \%$ ethanol and quantified by measuring $\mathrm{OD}_{590}$.

We examined attachment of GFP-labelled P. tunicata wild-type and RFP-labelled SM5 cells to microcrystalline cellulose. Attachment was monitored essentially as described by Bayer et al. (1983) and by direct confocal laser scanning microscopic (CLSM) imaging of fluorescent cells attached to cellulose particles. Cells grown using glucose or cellobiose were harvested after $24 \mathrm{~h}$ and cells grown using cellulose were harvested after $48 \mathrm{~h}$. The cells were then gently washed twice with PBS (centrifugation at $5000 \mathrm{~g}$ for $10 \mathrm{~min}$ ) and resuspended in $10 \mathrm{ml}$ PBS solution. The assay mixture consisted of $1 \mathrm{ml}$ cells $\left(\sim 10^{7}\right.$ cells $\left.\mathrm{ml}^{-1}\right)$ (pre-grown in glucose, cellobiose or microcrystalline cellulose), $1 \mathrm{ml} 20 \%$ microcrystalline cellulose in PBS and $1 \mathrm{ml}$ PBS. The mixture was shaken slowly for $1 \mathrm{~h}$ and incubated statically for $1 \mathrm{~h}$ at room temperature. The $\mathrm{OD}_{400}$ of the suspension was measured and compared to a control (identical cell suspension in PBS). To test the effect of mannose in the attachment of cells to cellulose, the attachment assay mixture was added with $100 \mathrm{mM} \alpha$-methyl-Dmannoside. The attachment assay for each strain was carried out in three replicates. The samples were mounted on a glass microscope slide and transmission fluorescent images were captured using an Olympus LSMGB200 confocal laser scanning microscope.
Preparation of axenic thallus of green alga $U$. australis and culture conditions. Axenic thallus of $U$. australis was obtained following the protocol of Rao et al. (2006). Briefly, plants of $U$. australis were collected from rocks at Clovelly Bay, Sydney, Australia. The collected plants were rinsed with $50 \mathrm{ml}$ autoclaved sea water and thallus discs of around $0.6 \mathrm{~cm}$ diameter were excised from the lower part of $U$. australis by using a steel punch. To remove other bacteria from the surface of $U$. australis, surfaces of $U$. australis discs were swabbed with sterile cotton tips and exposed to $0.012 \%(\mathrm{v} / \mathrm{v}) \mathrm{NaOCl}$ for $5 \mathrm{~min}$. Plant pieces were incubated in an antibiotic mixture containing $300 \mathrm{mg}$ ampicillin $\mathrm{l}^{-1}, 30 \mathrm{mg}$ polymyxin $\mathrm{l}^{-1}$ and $60 \mathrm{mg}$ gentamicin $1^{-1}$ for $24 \mathrm{~h}$ followed by $1 \mathrm{~h}$ recovery in $20 \mathrm{ml}$ sterile sea water.

Attachment assay with axenic $\boldsymbol{U}$. australis. GFP-tagged $P$. tunicata wild-type and SM5 mutant strains were grown on VNSS medium containing chloramphenicol for $24 \mathrm{~h}$. After $24 \mathrm{~h}$ the cell suspensions were centrifuged at $6000 \mathrm{~g}$ for $7 \mathrm{~min}$. The bacterial cells were rinsed twice with $10 \mathrm{ml}$ PBS and resuspended in PBS to an $\mathrm{OD}_{610}$ of $0 \cdot 35-0 \cdot 45$. The assay mixture consisted of $1 \mathrm{ml}$ washed cells, one axenic thallus disc of $U$. australis and $1 \mathrm{ml}$ PBS. The mixture was incubated for a period of $2 \mathrm{~h}$ with slow shaking and for $1 \mathrm{~h}$ without shaking at room temperature. The $U$. australis pieces were rinsed twice with $10 \mathrm{ml}$ PBS to remove unattached cells. For each sample, 10 images were manually analysed by counting the bacteria attached to the surface using CLSM. The attachment assay was repeated four times with three replicates for each bacterial strain.

\section{RESULTS}

\section{The $\boldsymbol{P}$. tunicata genome encodes a putative MSHA biogenesis locus}

DNA sequencing of the regions flanking the $\mathrm{Tn} 10$ insert in the $P$. tunicata SM5 mutant yielded a $4291 \mathrm{bp}$ nucleotide sequence with six potential ORFs (MshI1-MshM). Analysis of this region in the draft genome sequence of $P$. tunicata revealed an additional 11 ORFs predicted to be in two transcriptional units (Fig. 1). Promoter prediction analysis revealed potential transcriptional start sites approximately 19 bp upstream of the mshI1 start codon $(-10$ box 'ATTTAAGAT' and -35 box 'TTGCTT') and $34 \mathrm{bp}$ upstream of the $m s h B$ start codon $(-10$ box 'CAGTTAAAT' and -35 box 'TTGTAT'). These regions are in agreement with the -10 and -35 regions of the $E$. coli $\sigma^{70}$ promoter and match closely with other predicted promoter sequences for P. tunicata (Egan et al., 2002a). Following the translational stop of ORF17 $(m s h)$ is a region indicative of a transcriptional terminator ( $92 \mathrm{bp}$ downstream of $m s h Q$ ) (Mathews \& van Holde, 1990). The deduced amino acid sequence of the 17 ORFs showed homology to the secretory and structural genes for the MSHA pilus of various bacteria (data not shown). Moreover, the operon structure reflects that of the $V$. cholerae MSHA biogenesis locus with the only major difference being in the genes encoding the MshI protein. In $P$. tunicata there are two genes with similarity to different regions of the $V$. cholerae mshI gene and these have been termed mshI1 and mshI2, respectively (Fig. 1).

\section{Electron microscopic detection of cell surface pili}

Following the identification of the putative MSHA pilus operon, we examined whether $P$. tunicata expresses 


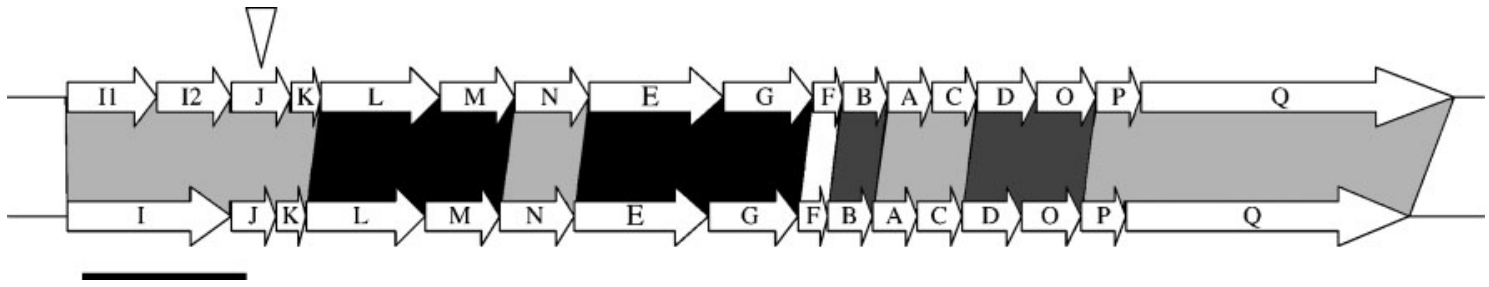

Fig. 1. Schematic representation of the predicted MSHA gene locus in P. tunicata (top) and $V$. cholerae El Tor (bottom). The entire locus is $17525 \mathrm{bp}$ in length and consists of 17 continuous ORFs (msh/1/2JKLMNEGFBACDOPQ). The scale bar represents approximately $2 \mathrm{~kb}$. The inverted triangle indicates the location of the transposon in the P. tunicata SM5 MSHA mutant strain. Black shading, $>45 \%$ identity; dark grey, 35-45\% identity; pale grey, 25-35\% identity; white, $<25 \%$ identity.

functional pili. Transmission electron micrographs of $P$. tunicata wild-type and the SM5 mutant cells are shown in Fig. 2. It was observed that $P$. tunicata wild-type cells are surrounded by flexible pili on their surface, each with a diameter of 7-8 $\mathrm{nm}$ and a length of $90-150 \mathrm{~nm}$. The mean number of surface pili per cell \pm SEM was calculated on 50 randomly selected cells. $P$. tunicata wild-type cells showed a mean number of surface pili per cell of $11 \cdot 32 \pm 0 \cdot 7$. $P$. tunicata SM5 mutant cells were non-piliated, confirming that the putative MSHA biogenesis operon is required for pilus expression. In addition, we observed that wild-type cells grown using cellobiose or cellulose as the sole carbon source exhibited a significant increase in the mean number of pili per cell, $14 \cdot 42 \pm 0 \cdot 2$ and $17 \cdot 22 \pm 0 \cdot 9$ respectively, than those grown in VNSS $(P<0 \cdot 0001)$.

\section{Assay for agglutination of red blood cells}

To further characterize the $P$. tunicata pilus, haemagglutination assays were performed. It was found that $P$. tunicata wild-type cells cause agglutination of horse red blood cells. Haemagglutination was observed when either exponentialor stationary-phase wild-type $P$. tunicata cells were used (Table 1). In contrast there was no haemagglutination observed in the presence of the SM5 mutant when cells were assayed during exponential phase; however, in stationaryphase cells, a low level of haemagglutination was observed. Addition of mannose to the assay mixture blocked haemagglutination by $P$. tunicata wild-type cells.

\section{Pili promote the attachment of $\boldsymbol{P}$. tunicata to abiotic surfaces}

Polystyrene microtitre plates, widely used in bacterial attachment assays (O’Toole \& Kolter, 1998; Taylor et al., 2002), were used as an abiotic test surface. After $1 \mathrm{~h}, P$. tunicata wild-type cells demonstrated considerably higher attachment compared to SM5 mutant cells as detected by the crystal violet staining of attached cells to wells of a polystyrene microtitre plate. The crystal-violet-stained $P$. tunicata wild-type cells displayed an $\mathrm{OD}_{590}$ of $0 \cdot 97 \pm 0 \cdot 09$, which was $2 \cdot 8$-fold higher than the crystal-violet-stained SM5 mutant cells $(0 \cdot 34 \pm 0 \cdot 01)$.
The attachment of $P$. tunicata wild-type and SM5 mutant cells to cellulose was also tested. Cellulose is a major surface polymer of C. intestinalis (De Leo et al., 1977) and $U$. australis (Chapman, 1979), and P. tunicata is frequently isolated from these surfaces. $P$. tunicata wild-type cells (grown with glucose as the carbon source) demonstrated
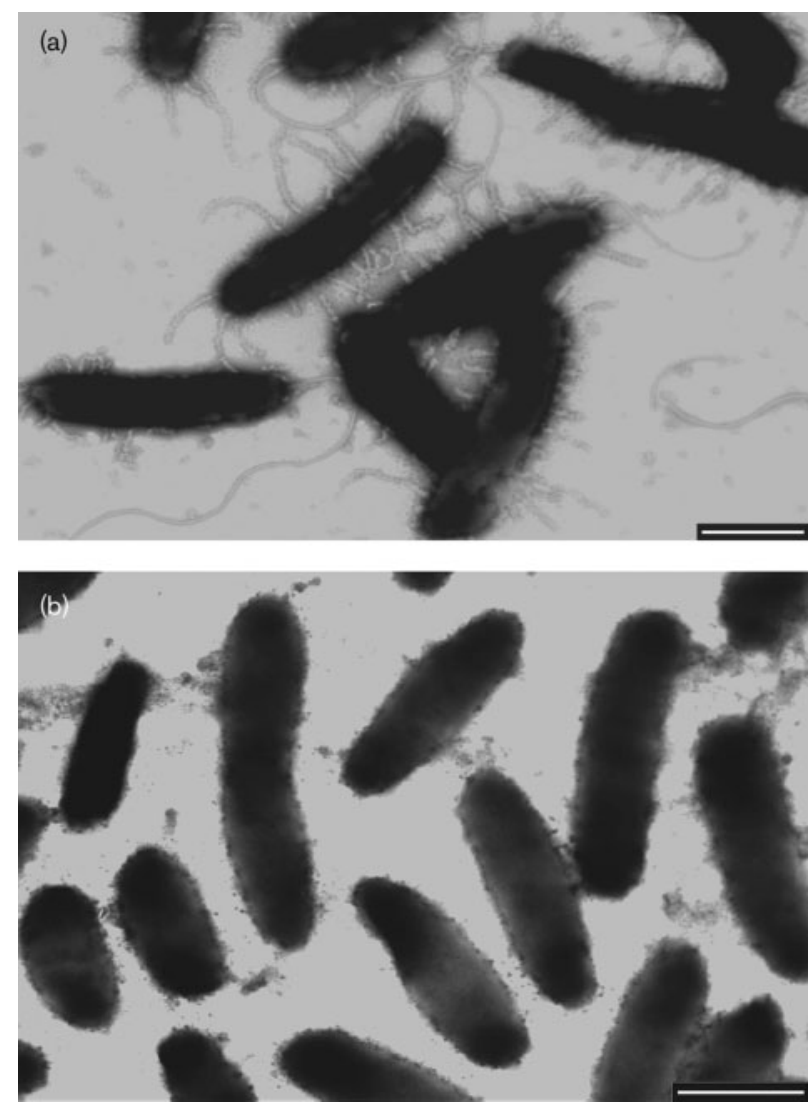

Fig. 2. Transmission electron micrographs showing (a) $P$. tunicata wild-type cells expressing pili on the surface, and (b) $P$. tunicata SM5 mutant cells displaying the non-piliated phenotype. Both strains were grown on VNSS agar plates for $24 \mathrm{~h}$. All samples were negatively stained with $2 \%$ phosphotungstic acid. Bars, $1 \mu \mathrm{m}$. 
Table 1. Haemagglutination of horse erythrocytes by bacteria at different growth stages

Values represent the reciprocal of the dilution in which haemagglutination can be observed. The results of the assay performed in the presence of $1 \% \alpha$-methyl-D-mannoside were zero for all strains tested in both growth phases.

\begin{tabular}{|lcc|}
\hline Bacterial strain & Stationary phase & Exponential phase \\
\hline Vibrio cholerae M1615 ( + control) & $>50$ & $>50$ \\
P. tunicata wild-type & 20 & 20 \\
P. tunicata msh (SM5) mutant & $<2$ & 0 \\
\hline
\end{tabular}

attachment to microcrystalline cellulose which resulted in a $22.2 \%$ reduction in the final $\mathrm{OD}_{400}$ of the assay mixture (Fig. 3). SM5 mutant cells, however, attached less effectively to cellulose showing only $4 \%$ reduction. Moreover, attachment of wild-type $P$. tunicata was enhanced when cells were pre-grown on cellulose and cellobiose $(29 \cdot 4$ and $40 \cdot 7 \%$ reduction, respectively). SM5 mutant cells pregrown in cellobiose and cellulose showed $26 \cdot 7$ and $14 \cdot 5 \%$ reduction of the final $\mathrm{OD}_{400}$ of the assay mixture, respectively. It was also demonstrated that mannose significantly inhibits attachment of $P$. tunicata wild-type cells to cellulose, displaying only $3 \cdot 7 \%$ reduction of the final $\mathrm{OD}_{400}$ of the attachment assay mixture.

We also compared the attachment of the two strains to cellulose using fluorescently tagged cells and CLSM. Transmission fluorescence images revealed that substantial numbers of $P$. tunicata wild-type cells attached to microcrystalline cellulose (Fig. 4b). In contrast, the SM5 mutant

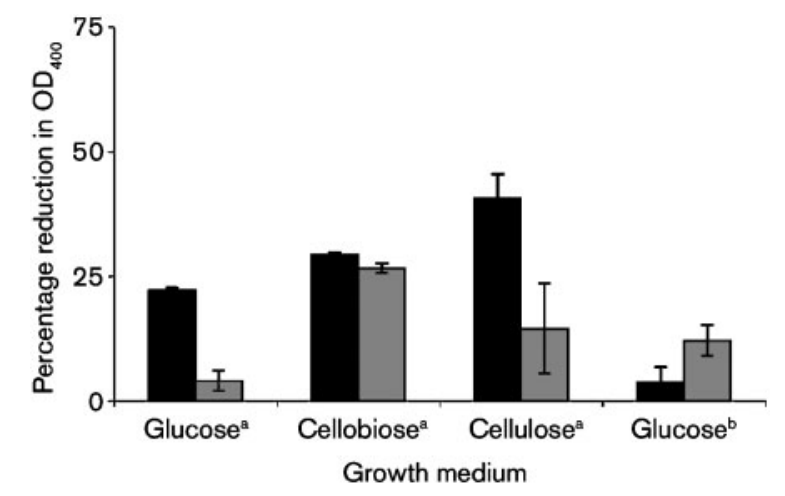

Fig. 3. Attachment of $P$. tunicata wild-type (black bars) and SM5 mutant (grey bars) to microcrystalline cellulose (Avicel). The microcrystalline cellulose was mixed with bacterial cells $\left(\sim 10^{7}\right.$ c.f.u. $\left.\mathrm{ml}^{-1}\right)$ for $1 \mathrm{~h}$ and the final $\mathrm{OD}_{400}$ of the cellulose and bacterial cell suspension was measured after $1 \mathrm{~h}$ incubation. The attachment of bacteria to cellulose was scored by the reduction in the final $\mathrm{OD}_{400}$ of the suspension. ${ }^{\mathrm{a}}$, Bacteria were grown in this medium prior to the attachment assay which was performed in the absence of mannose; ${ }^{b}$, bacteria were grown in this medium prior to the attachment assay which was performed in the presence of mannose. Error bars represent the SEM of triplicate cultures. cells attached less to cellulose both during attachment in pure culture and mixed with wild-type $P$. tunicata cells (Fig. 4c and a, respectively).

\section{Pili promote attachment of $P$. tunicata to the surface of the green alga $\boldsymbol{U}$. australis}

Axenic discs of $U$. australis were prepared and resulted in over $90 \%$ reduction of the natural microbial community as previously demonstrated (Rao et al., 2006). Axenic $U$. australis thallus discs were incubated with $P$. tunicata wildtype: GFP and P. tunicata SM5: GFP cells, and attachment assays were carried out in PBS, $\mathrm{pH} 7 \cdot 4$. The total cell counts per $\mathrm{mm}^{2}$ of algal surface for $P$. tunicata wild-type and the SM5 mutant were $2377 \pm 607$ and $996 \pm 362$ cells, respectively. $P$. tunicata wild-type cells demonstrated $2 \cdot 4$-fold enhanced attachment compared to the SM5 mutant on the surface of $U$. australis (Fig. 5).

\section{DISCUSSION}

Surface-associated bacteria on marine sessile organisms may provide benefits to their hosts. For example, P. tunicata produces a range of inhibitory compounds and may offer protection against colonization of biofouling and hostpathogenic organisms (Egan et al., 2000, 2001b; Holmström et al., 1992; James et al., 1996). Such interactions are initiated by the attachment and initial colonization of bacteria to the host surface, and may be facilitated by the expression of surface adhesins. In $V$. cholerae El Tor, the attachment to phytoplankton and zooplankton surfaces is mediated by the MSHA pilus (Chiavelli et al., 2001). Recently, it was reported that an MSHA pilus promotes interaction between $V$. cholerae El Tor and haemolymph of the mussel Mytilus galloprovincialis (Zampini et al., 2003). In this study, we investigated the role of an MSHA-like pilus in the attachment of $P$. tunicata to the surface of $U$. australis, a living surface from which $P$. tunicata has been isolated in the marine environment.

This study describes a gene locus proposed to be involved in the export and assembly of an MSHA pilus in P. tunicata. We have sequenced the DNA flanking a Tn10-disrupted ORF with high homology to mshJ of $V$. cholerae (Marsh \& Taylor, 1999), and together with analysis of the flanking region in the recently obtained draft genome of $P$. tunicata, revealed 17 contiguous ORFs (mshIII2JKLMNEGFBACDOPQ) with 

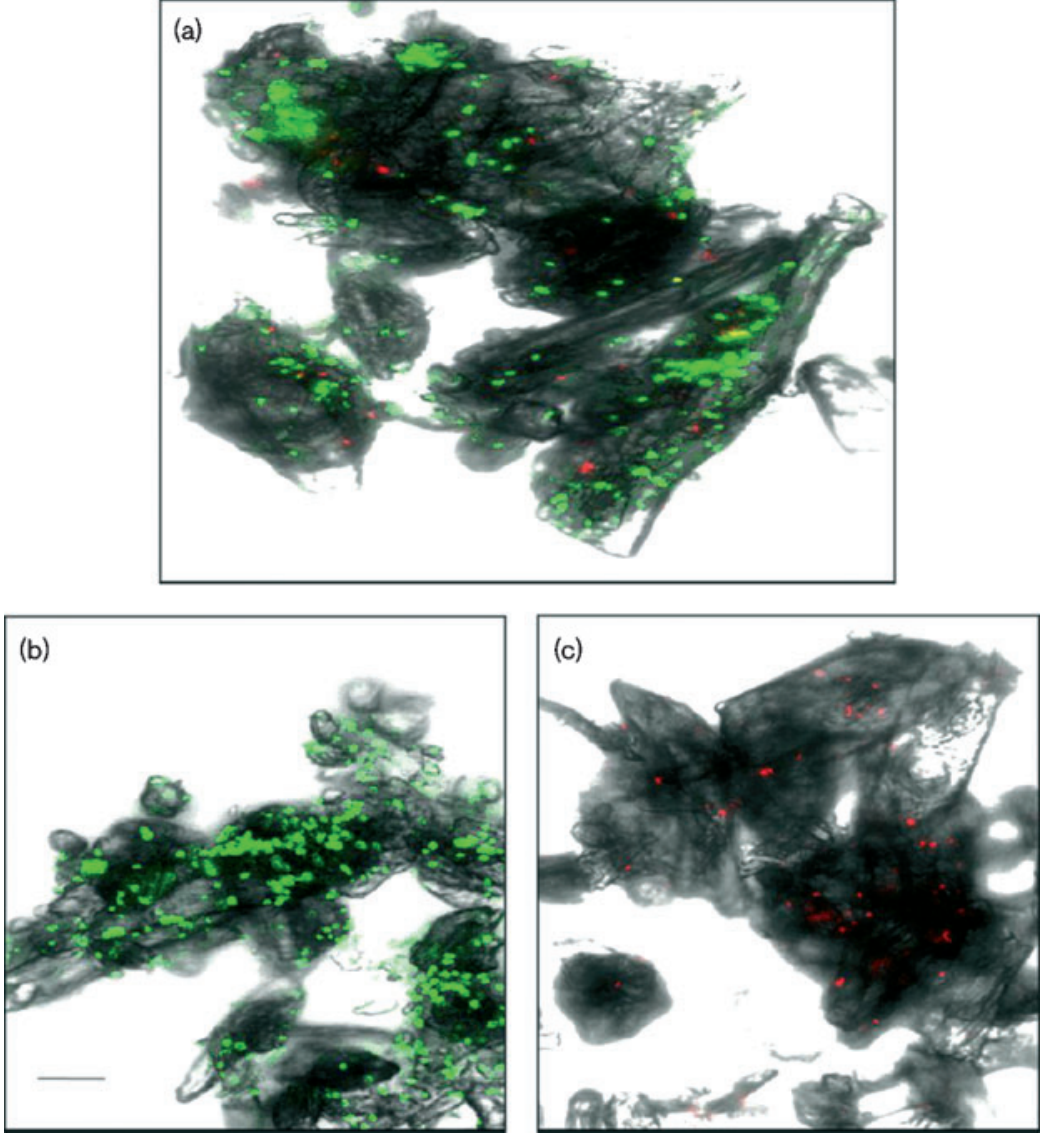

Fig. 4. Transmission fluorescent micrographs using CLSM showing attachment of bacterial strains to microcrystalline cellulose (Avicel) after the adherence assay. (a) $P$. tunicata wildtype: GFP and SM5 mutant: RFP added to the plant as a $1: 1$ mixture; (b) $P$. tunicata wild-type : GFP only and (c) SM5 mutant:RFP only. Bar, $20 \mu \mathrm{m}$. similar genetic organization and high homology to MSHA pili biogenesis and secretory proteins of various Gramnegative bacteria, including Pseudoalteromonas haloplanktis, Shewanella baltica, V. cholerae, Vibrio parahaemolyticus and Vibrio vulnificus. The best characterized MSHA pilus gene locus is that of $V$. cholerae El Tor (Marsh \& Taylor, 1999). The 17 P. tunicata ORFs identified in this study share major similarities with the MSHA pili secretory and structural genes of $V$. cholerae, including (1) homology of the predicted amino acid sequences, (2) similarity of the predicted location of the gene products, (3) similarity in their organization, orientation and arrangement, and (4) the presence of polycistronic genes, where overlapping stop and start codons of ORFs have been identified.

TEM studies revealed the presence of flexible pili on the cell surface of $P$. tunicata, with ultrastructural characteristics similar to pili of other Gram-negative bacteria. In contrast, the P. tunicata SM5 mutant, disrupted in the MSHA gene locus, showed no expression of pili on the cell surface. Biogenesis of pili requires numerous gene products, including a structural prepilin subunit, ancillary proteins with prepilin-like leader sequences, inner- and outermembrane proteins and nucleotide-binding proteins (Alm \& Mattick, 1997). It has been reported that a mutation in any of these genes is sufficient to prevent the assembly of functional pili (Strom \& Lory, 1993). For example, mutation of any one of the secretory genes pilO, pilP or pilQ in
Pseudomonas aeruginosa resulted in loss of pili, confirming the importance of these mutated genes in pilus biogenesis (Martin et al., 1995). These secretory genes belong to an operon for secretion and export which includes two other genes (pilM and pilN) required in the biogenesis of fimbriae in Pseudomonas aeruginosa (Martin et al., 1995). In $V$. cholerae, it has been reported that the expression of MSHA to form functional pili on the bacterial surface is completely dependent on the transcription and expression of two operons: secretory and structural (Martin et al., 1995). Deletion in any of the putative promoter regions upstream of $m s h I$, a secretory gene, or $m s h B$, a structural gene, abolished MSHA pilus assembly, secretion and expression (Martin et al., 1995). Additionally, mutation in $m s h E$, a secretory gene in the MSHA biogenesis locus, showed abolished haemagglutination (Hase et al., 1994). The fact that the $P$. tunicata SM5 mutant displayed a non-piliated phenotype and was unable to mediate haemagglutination suggests that the MSHA gene locus is required for pilus biogenesis in P. tunicata.

Gram-negative bacteria bind to surfaces via the tip adhesins of the pili (Strom \& Lory, 1993). These adhesins may have different specific receptors. For example, V. cholerae El Tor strains express a haemagglutinin pilus with a preference for mannose receptors (Jonson et al., 1991). We have demonstrated that the $P$. tunicata pilus causes haemagglutination of horse red blood cells. As for $V$. cholerae El Tor, the 

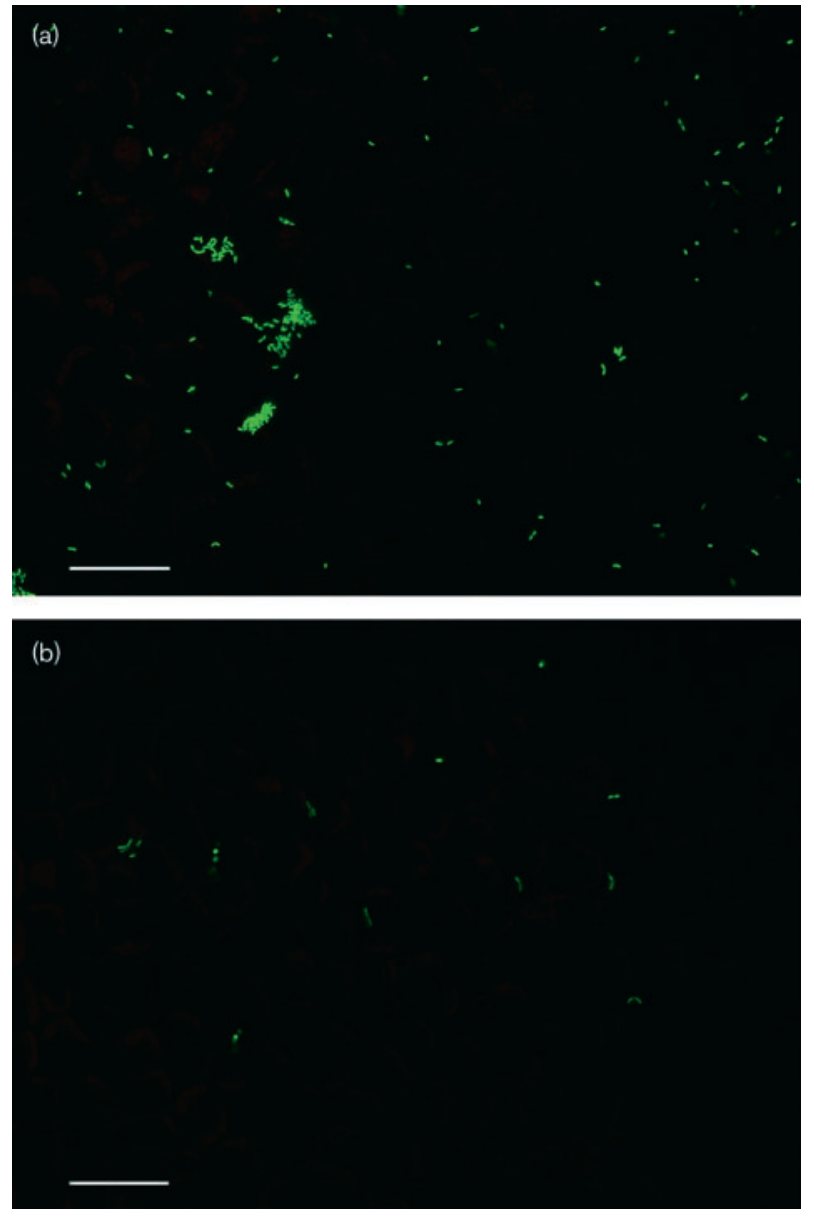

Fig. 5. CLSM images of (a) P. tunicata wild-type GFP and (b) $P$. tunicata (SM5) mshJ mutant GFP attached to the surface of U. australis. Bars, $50 \mu \mathrm{m}$.

clumping of red blood cells was abolished in the presence of mannose.

The MSHA pili expressed by $V$. cholerae cells are known to be involved in the attachment to and colonization of surfaces. The MSHA pilus is an important attachment factor in aquatic environments and has been shown to mediate attachment to solid substrates (Chiavelli et al., 2001; Watnick et al., 1999). It was reported that the $V$. cholerae El Tor MSHA mutant strain is unable to form biofilms and shows decreased adherence to abiotic and biotic surfaces (Watnick et al., 1999). In this study, we first tested attachment of $P$. tunicata wild-type and SM5 mutant cells to polystyrene and to cellulose, the surface polymer of $U$. australis and $C$. intestinalis from which $P$. tunicata has been isolated. The SM5 mutant cells showed less attachment to both polystyrene and cellulose surfaces, in comparison with the piliated wild-type strain. This suggests a key role for the $P$. tunicata MSHA pilus in the attachment to both surfaces.

To determine if the MSHA pilus is involved in mediating the attachment of $P$. tunicata cells to living surfaces we compared the ability of the piliated and non-piliated strains to attach directly to the surface of the green alga $U$. australis. The attachment assays revealed significantly higher numbers of attached P. tunicata wild-type cells compared to SM5 mutant cells at the surface of $U$. australis. However, a small number of SM5 cells did attach to the algal surface, suggesting that other mechanisms may be involved in the adhesion of $P$. tunicata to the surface of $U$. australis (for example non-specific physicochemical interactions between the bacterial cell and plant surface). Nevertheless, our findings indicate that the MSHA pilus is a major determinant for attachment of $P$. tunicata to $U$. australis.

This study also showed that specific growth conditions affect the expression of the P. tunicata MSHA pilus. P. tunicata cells grown in cellulose or cellobiose were found to be hyperpiliated when examined under TEM. These specific substrates also promote attachment of $P$. tunicata to cellulose. We observed that wild-type cells pregrown in cellulose or cellobiose displayed enhanced attachment to cellulose compared to cells grown on other carbon sources (a smaller increase in adhesion to cellulose of the SM5 mutant grown in cellulose or cellobiose was also observed, although the reason for this is unclear). These studies indicate that cellulose or cellobiose may serve as environmental signals which induce expression of the MSHA pilus and thus promote attachment of $P$. tunicata to cellulose-containing surfaces. Our findings concur with previous reports that chemosensory mechanisms can control pilus expression. In Pseudomonas aeruginosa, expression of pilA is controlled by a two-component sensorregulator gene pair, pilS and pilR (Hobbs et al., 1993). The PilS protein is a sensor protein located upstream of the regulator protein PilR, thought to be responsible for sensing unknown environmental signals (Boyd, 2000). In V. cholerae, the response of tcp (toxin-coregulated pili) genes to environmental stimuli is mediated by the ToxR regulon (Strom \& Lory, 1993). The ToxR protein is a major sensor and regulator protein that transmits signals from the periplasmic side of the inner membrane and regulates transcription of virulence factors, including the toxincoregulated pili. Interestingly, a putative transcriptional regulator, WmpR with homology to ToxR has been identified in P. tunicata (Egan et al., 2002b). This gene is involved in the regulation of the expression of antifouling compounds in $P$. tunicata (Egan et al., 2002b). The P. tunicata $w m p R$ mutant is devoid of pili on its surface when examined using TEM (data not shown). It is possible that the regulatory activity of $w m p R$ may explain the surface-sensing mechanisms demonstrated by $P$. tunicata in response to environmental stimuli. In the marine ecosystem, the $P$. tunicata $w m p R$ gene product may sense an environmental signal (e.g. cellulose or surface polymers of $C$. intestinalis and $U$. australis) and respond by increasing the expression of MSHA pili on the cell surface, hence promoting the attachment of $P$. tunicata to the surfaces of these marine organisms.

In summary, we have identified a gene locus (mshI1I2JKLMNEGFBACDOPQ) with similar genetic 
organization and high homology to MSHA pilus biogenesis operons of marine vibrios, and have shown that the msh gene locus is required for the expression of a MSHA pilus on the $P$. tunicata cell surface. Our findings demonstrate that production of the MSHA pilus mediates the attachment of $P$. tunicata to surfaces and that this pilus is a key determinant of attachment of $P$. tunicata to both abiotic and living surfaces. We also provide evidence that expression of MSHA pilus is enhanced in the presence of cellulose or cellobiose and propose that substrate-sensing mechanisms and pilus expression in $P$. tunicata have a profound effect on the ecological distribution of this bacterium in marine surface ecosystems.

\section{ACKNOWLEDGEMENTS}

This work was supported by the Australian Research Council and by the Centre for Marine Biofouling and Bio-Innovation, University of New South Wales, Australia.

\section{REFERENCES}

Alm, R. A. \& Mattick, J. S. (1997). Genes involved in the biogenesis and function of type-4 fimbriae in Pseudomonas aeruginosa. Gene 192, 89-98.

Altschul, S. F., Gish, W., Miller, W., Meyers, E. W. \& Lipman, D. J. (1990). Basic Local Alignment Search Tool. J Mol Biol 215, 403-410.

Bagdasarian, M., Lurz, R., Ruckert, B., Franklin, F. C., Bagdasarian, M. M., Frey, J. \& Timmis, K. N. (1981). Specific-purpose plasmid cloning vectors. II. Broad host range, high copy number, RSF1010derived vectors, and a host-vector system for gene cloning in Pseudomonas. Gene 16, 237-247.

Bayer, E., Kenig, R. \& Lamed, R. (1983). Adherence of Clostridium thermocellum to cellulose. J Bacteriol 156, 818-827.

Boyd, J. M. (2000). Localization of the histidine kinase PilS to the poles of Pseudomonas aeruginosa and identification of a localization domain. Mol Microbiol 36, 153-162.

Bryers, J. \& Characklis, W. (1982). Processes governing primary biofilm formation. Biotechnol Bioeng 24, 2451-2476.

Chapman, A. R. O. (1979). Biology of Seaweeds: Levels of Organization. Baltimore, MD: University Park Press.

Chiavelli, D. A., Marsh, J. W. \& Taylor, R. K. (2001). The mannosesensitive hemagglutinin of Vibrio cholerae promotes adherence to zooplankton. Appl Environ Microbiol 67, 3220-3225.

Cormack, B. P., Valdivia, R. H. \& Falkow, S. (1996). FACS-optimized mutants of the green fluorescent protein (GFP). Gene 173, 33-38.

De Leo, G., Patricolo, E. \& D'Ancona-Lunetta, G. (1977). Studies on the fibrous components of the test of Ciona intestinalis Linnaes. Cellulose-like polysaccharide. Acta Zoo 58, 135-141.

de Nys, R., Steinberg, P., Willemsen, P., Dworjanyn, S., Gabelish, C. \& King, R. (1995). Broad spectrum effects of secondary metabolites from the red alga Delisea pulchra in antifouling assays. Biofouling $\mathbf{8}$, 259-271.

Egan, S., Thomas, T., Holmström, C. \& Kjelleberg, S. (2000). Phylogenetic relationship and antifouling activities of bacterial epiphytes from the marine alga Ulva lactuca. Environ Microbiol 2, 343-347.
Egan, S., Holmström, C. \& Kjelleberg, S. (2001a). Pseudoalteromonas ulvae sp. nov., a bacterium with antifouling activities isolated from the surface of a marine alga. Int J Syst Evol Microbiol 51, 1499-1504.

Egan, S., James, S., Holmström, C. \& Kjelleberg, S. (2001b). Inhibition of algal spore germination by the marine bacterium Pseudoalteromonas tunicata. FEMS Microbiol Ecol 35, 67-73.

Egan, S., James, S., Holmström, C. \& Kjelleberg, S. (2002a). Correlation between pigmentation and antifouling compounds produced by Pseudoalteromonas tunicata. Environ Microbiol 4, 433-442.

Egan, S., James, S. \& Kjelleberg, S. (2002b). Identification and characterization of a putative transcriptional regulator controlling the expression of fouling inhibitors in Pseudoalteromonas tunicata. Appl Environ Microbiol 68, 372-378.

Gardel, C. \& Mekalanos, J. (1996). Alterations in Vibrio cholerae motility phenotypes correlate with changes in virulence factor expression. Infect Immun 64, 2246-2255.

Harrison, P. (1992). Control of microbial growth and of amphipod gazing by water-soluble compounds from leaves of Zostera marina. Mar Biol 67, 25-30.

Hase, C. C., Bauer, M. E. \& Finkelstein, R. A. (1994). Genetic characterization of mannose-sensitive hemagglutinin (MSHA)-negative mutants of Vibrio cholerae derived by Tn 5 mutagenesis. Gene 150, 17-25.

Hobbs, M., Collie, E., Free, P., Livingston, S. \& Mattick, J. (1993). PilS and PilR, a two component transcriptional regulatory system controlling expression of type 4 fimbriae in Pseudomonas aeruginosa. Mol Microbiol 7, 669-682.

Holmström, C. \& Kjelleberg, S. (1999). Marine Pseudoalteromonas species are associated with higher organisms and produce biologically active extracellular agents. FEMS Microbiol Ecol 30, 285-293.

Holmström, C., Rittschof, D. \& Kjelleberg, S. (1992). Inhibition of settlement by larvae of Balanus amphitrite and Ciona intestinalis by a surface-colonizing marine bacterium. Appl Environ Microbiol 58, 2111-2115.

Holmström, C., James, S., Egan, S. \& Kjelleberg, S. (1996). Inhibition of common fouling organisms by marine bacterial isolates with special reference to the role of pigmented bacteria. Biofouling 10, 251-259.

Holmström, C., James, S., Neilan, B., White, D. \& Kjelleberg, S. (1998). Pseudoalteromonas tunicata sp. nov., a bacterium that produces antifouling agents. Int J Syst Bacteriol 48, 1205-1212.

Holmström, C., Egan, S., Franks, A., McCloy, S. \& Kjelleberg, S. (2002). Antifouling activities expressed by marine surface associated Pseudoalteromonas species. FEMS Microbiol Ecol 41, 47-58.

James, S., Holmström, C. \& Kjelleberg, S. (1996). Purification and characterization of a novel antibacterial protein from the marine bacterium D2. Appl Environ Microbiol 62, 2783-2788.

Jonson, G., Holmgren, J. \& Svennerholm, A. (1991). Identification of a mannose-binding pilus on Vibrio cholerae El Tor. Microb Pathog 11, 433-441.

Marden, P., Tunlid, A., Malmcrona-Friberg, K., Odham, G. \& Kjelleberg, S. (1985). Physiological and morphological changes during short term starvation of marine bacterial isolates. Arch Microbiol 142, 326-332.

Marsh, J. W. \& Taylor, R. K. (1999). Genetic and transcriptional analyses of the Vibrio cholerae mannose-sensitive hemagglutinin type 4 pilus gene locus. J Bacteriol 181, 1110-1117.

Martin, P., Watson, A., McCaul, T. \& Mattick, J. (1995). Characterization of a five-gene cluster required for the biogenesis of type 4 fimbriae in Pseudomonas aeruginosa. Mol Microbiol 16, 497-508.

Mary, A., Mary, V., Rittschof, D. \& Nagabhushanam, R. (1993). Bacterial-barnacle interaction: potential for using juncellins and 
antibiotics to alter structure of bacterial communities. J Chem Ecol 19, 2155-2167.

Mathews, C. K. \& van Holde, K. E. (1990). Biochemistry. Redwood City, CA: The Benjamin/Cummings Publishing Company.

Maximilien, R., de Nys, R., Holmström, C., Gram, L., Givskov, M., Crass, K., Kjelleberg, S. \& Steinberg, P. (1995). Chemical mediation of bacterial surface colonization by secondary metabolites from red algal Delisea pulchra. Aquat Microb Ecol 15, 233-246.

Morand, P. C., Tattevin, P., Eugene, E., Beretti, J.-L. \& Nassif, X. (2001). The adhesive property of the type IV pilus-associated component PilC1 of pathogenic Neisseria is supported by the conformational structure of the N-terminal part of the molecule. Mol Microbiol 40, 846-856.

Neidhardt, F., Bloch, P. \& Smith, D. (1974). Culture medium for enterobacteria. J Bacteriol 119, 736-747.

O'Toole, G. A. \& Kolter, R. (1998). Flagellar and twitching motility are necessary for Pseudomonas aeruginosa biofilm development. Mol Microbiol 30, 295-304.

Rao, D., Webb, J. S. \& Kjelleberg, S. (2005). Competitive interactions in mixed-species biofilms containing the marine bacterium Pseudoalteromonas tunicata. Appl Environ Microbiol 71, 1729-1736.

Rao, D., Webb, J. S. \& Kjelleberg, S. (2006). Microbial colonization and competition on the marine alga Ulva australis. Appl Environ Microbiol 72, 5547-5555.
Reese, M. (2001). Application of a time-delay neural network to promoter annotation in the Drosophila melanogaster genome. Comp Chem 26, 51-56.

Strom, M. S. \& Lory, S. (1993). Structure-function and biogenesis of the type IV pili. Annu Rev Microbiol 47, 565-596.

Taylor, C. M., Beresford, M., Epton, H. A. S., Sigee, D. C., Shama, G., Andrew, P. W. \& Roberts, I. S. (2002). Listeria monocytogenes relA and $h p t$ mutants are impaired in surface-attached growth and virulence. J Bacteriol 184, 621-628.

Tillett, D. \& Neilan, B. A. (1999). n-Butanol purification of dye terminator sequencing reactions. Biotechniques 26, 606-608, 610.

Watnick, P. I. \& Kolter, R. (1999). Steps in the development of a Vibrio cholerae El Tor biofilm. Mol Microbiol 34, 586-595.

Watnick, P. I., Fullner, K. J. \& Kolter, R. (1999). A role for the mannose-sensitive hemagglutinin in biofilm formation by Vibrio cholerae El Tor. J Bacteriol 181, 3606-3609.

Zampini, M., Canesi, L., Betti, M., Ciacci, C., Tarsi, R., Gallo, G. \& Pruzzo, C. (2003). Role for mannose-sensitive hemagglutinin in promoting interactions between Vibrio cholerae El Tor and mussel hemolymph. Appl Environ Microbiol 69, 5711-5715.

Zolfaghar, I., Evans, D. J. \& Fleiszig, S. M. J. (2003). Twitching motility contributes to the role of pili in corneal infection caused by Pseudomonas aeruginosa. Infect Immun 71, 5389-5393. 